\title{
Body Height and Weight of Patients with Childhood Onset and Adult Onset Thyrotoxicosis
}

\author{
Junta TAKAMATSU, NaOfumi KOBE, Mitsuru ITO, AND NAKAAKI OHSAWA
}

First Department of Medicine, Osaka Medical College, Takatsuki 569-0801, Japan

\begin{abstract}
The present study has compared body height and weight of thyrotoxic female patients of childhood onset and adult onset. The body height of 141 out of 143 (99\%) adult-onset thyrotoxic patients was within the range of mean $\pm 2 S D$ for the age-matched general Japanese female population. On the other hand, in 42 patients with childhood-onset thyrotoxicosis, $6(14 \%)$ had their height being greater than the mean +2 SD of general population, and $34(81 \%)$ were taller than the mean value. In 86 patients with siblings, $42(49 \%)$ were at least $2 \mathrm{~cm}$ taller than their sisters, and $26(30 \%)$ were more than $2 \mathrm{~cm}$ shorter than their sisters. The body weight of 27 out of $42(68 \%)$ patients younger than 20 years was not decreased but was even greater than the mean value for the age-matched general population. The results indicate that excessive thyroid hormone in vivo enhances body height in humans. The increased body weight in some young patients suggests that enhanced dietary intake due to increased appetite in hyperthyroidism has overcome the energy loss with increased metabolism.
\end{abstract}

Key words: Body weight, Thyroid hormone, Hyperthyroidism, Growth, Height

(Endocrine Journal 46: S101-S103, 1999)

SHORT stature is a well known skeletal manifestation of thyroid hormone defects in children [1], but changes in body structure in response to excessive thyroid hormone have not been well studied. There is a report of five children with hyperthyroidism before and during antithyroid treatment, in whom the height age were all more than 3 standard deviations (SD) above the mean for normal children, but the bone age also was accelerated, so that the relationship of bone age to height age remained normal [2].

The growth hormone concentration in serum is reported to be low even though the production rate is increased [3], but, as far as we know, no exact data on body height in thyrotoxic patients have been reported in recent years. The present study has compared the height of thyrotoxic patients with adult onset and childhood onset. The study also has examined body weight, since it is

Correspondence to: Dr. Junta TAKAMATSU, First Department of Medicine, Osaka Medical College, 2-7, Daigakumachi, Takatsuki, Osaka, Japan believed that thyrotoxic patients have decreased body weight.

\section{Subjects and Methods}

One hundred and eighty-five Japanese female patients with thyrotoxic Graves' disease were studied. In 143 of them, thyrotoxicosis started at above the age of 20 , and the remaining 42 had the onset before 15 years old. Eighty-six patients had sisters older than 20 years. They were treated with antithyroid drugs, subtotal thyroidectomy or radioiodine, and most of them became euthyroid after the treatment. Body height and weight were measured with an accurate scale.

\section{Results}

As shown in Fig. 1A, the body height of 143 adult onset thyrotoxic patients was within range of the mean $\pm 2 \mathrm{SD}$ for the age-matched general 

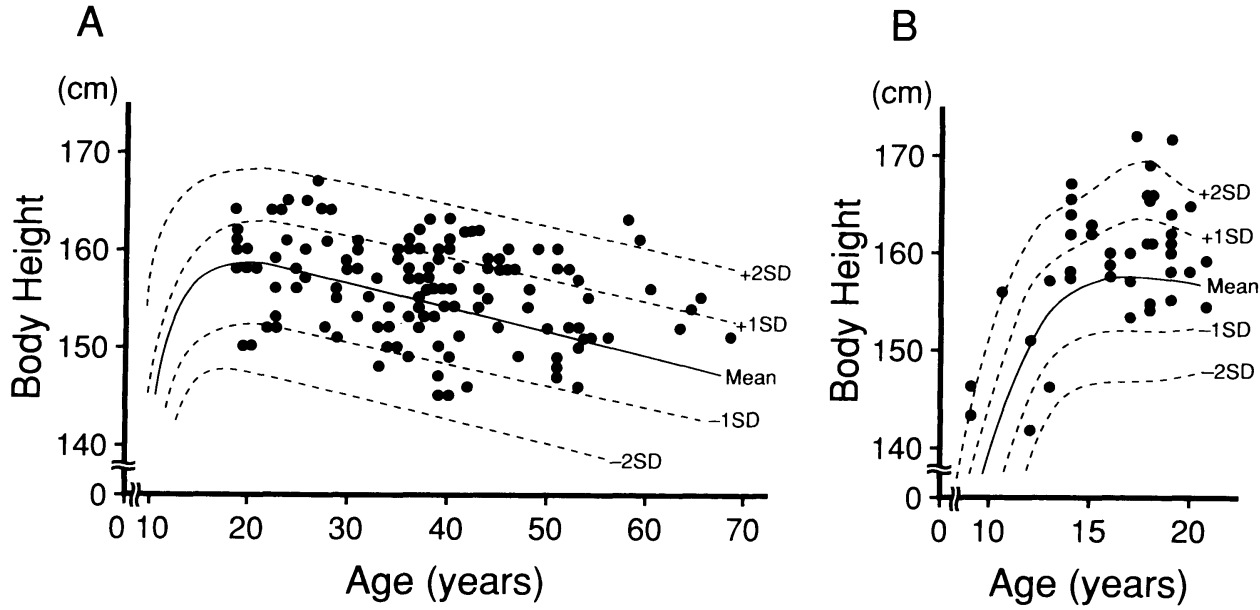

Fig. 1. Body height of patients with adult onset thyrotoxicosis (A) and childhood onset thyrotoxicosis (B). Individual patients' data are plotted. The solid line and dotted lines represent the mean value, and $\pm 1 \mathrm{SD}$ and $\pm 2 \mathrm{SD}$ of the general Japanese female population.

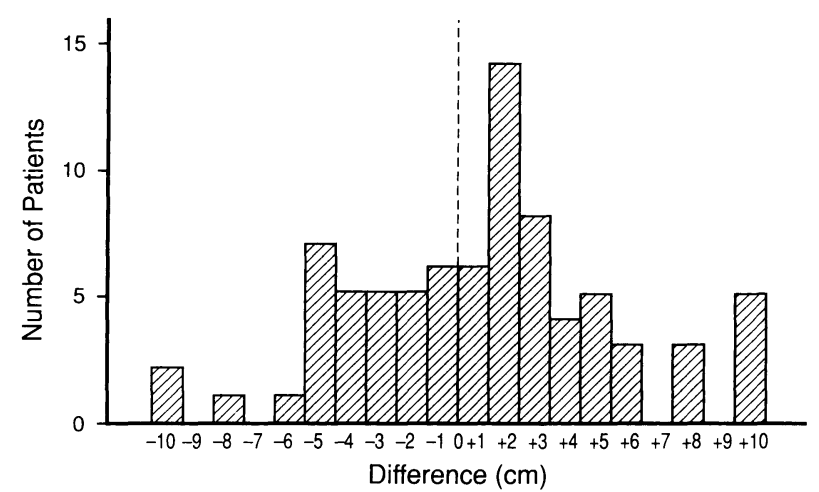

Fig. 2. Histogram of body height differences between thyrotoxic patients and their siblings. The plus signs indicate that the patients were taller than their sisters.

population of Japanese women in all except 2 patients $(1 \%)$ who were taller than the mean $+2 S D$. On the hand, as shown in Fig. 1B, 6 out of 42 patients (14\%) with childhood onset thyrotoxicosis were taller than the mean $+2 \mathrm{SD}$ for the general female population. In addition, 34 out of $42(81 \%)$ childhood onset thyrotoxic patients were taller than the mean for the general female population.

As shown in Fig. 2, the body height difference between an individual patient and her sister ranged from -10 to $+10 \mathrm{~cm}$, and in 18 of 86 patients (21\%) height differed less than $2 \mathrm{~cm}$ from that of their siblings. Forty-two (49\%) were more than $2 \mathrm{~cm}$ taller than their siblings, and the remaining 26 (30\%)

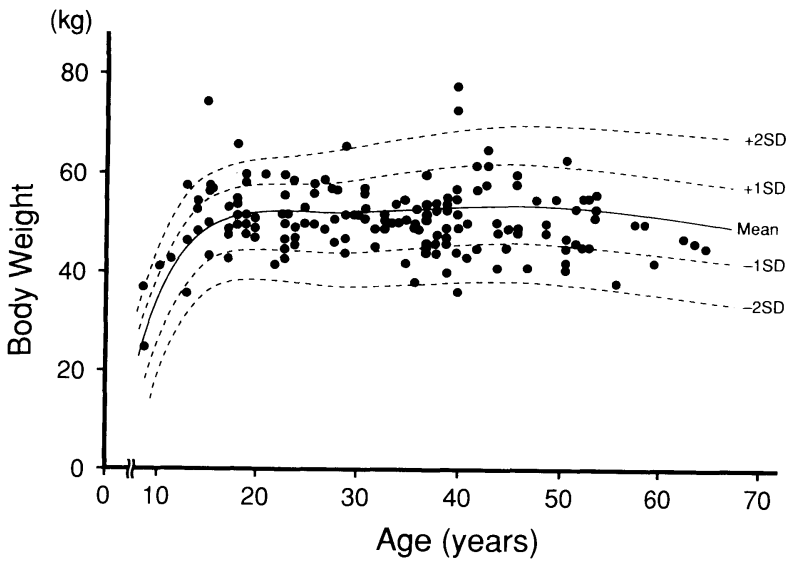

Fig. 3. Body weight of patients with thyrotoxicosis. Individual patients' data are plotted.

were more than $2 \mathrm{~cm}$ shorter than their sibling.

As shown in Fig. 3, the body weight of 105 patients older than 30 years tended to be decreased: 74 of them $(70 \%)$ had a body weight less than the mean value for most age-matched Japanese women. On the other hand, in 40 patients younger than 20 years, $27(68 \%)$ had a body weight being not less but even greater than the mean value for the general population.

\section{Discussion}

The increased body height of patients with 
childhood onset thyrotoxicosis suggests that excessive thyroid hormone in vivo enhances bone growth in humans. It is speculated that the effect of thyroid hormone is mediated not only by growth hormone or insulin-like growth factor-1 but also by some other factors, from our recent experience of growth hormone treatment of patients with resistance to thyroid hormone [4].
It seems unique that the body weight of thyrotoxic patients was not decreased but even increased, particularly in some young patients. This suggests that the enhanced dietary intake with increased appetite has overcome the energy loss with increased metabolism. Careful follow-up of body height and weight in childhood onset thyrotoxic patients may merit further investigation.

\section{References}

1. Delange FM (1996) Endemic cretinism. In: Braverman LE, Utiger RD (eds) The Thyroid. Lippincott-Raven, Philadelphia, 756-767.

2. Schlesinger S, Mac Gillivray MH, Munschauer RW (1973) Acceleration of growth and bone maturation in childhood thyrotoxicosis. Pediatrics 83: 233-239.

3. Taylor AL, Finster JL, Mintz DH (1969) Metabolic clearance and production rates of human growth hormone. J Clin Invest 48: 2349-2355.

4. Takamatsu J, Ito M, Yamano Y, Fukao T, Sakane S, Ohsawa N (1996) Defective response of body height to growth hormone treatment in resistance to thyroid hormone with short stature. Endocr J 43 (Suppl): 131-133. 